\title{
Non, la maladie de Chagas n'est pas le nouveau sida des Amériques !
}

\author{
J.-F. Pays \\ Reçu le 21 août 2012 ; accepté le 27 septembre 2012 \\ (C) Société de pathologie exotique et Springer-Verlag France 2012
}

\begin{abstract}
Résumé Maladie de Chagas et sida ne sauraient être associés, voire confondus sous un même vocable sans distordre la réalité, comme cela a été fait dans un article médical récent intitulé : Chagas disease: "The New HIV/AIDS of the Americas". Si la maladie de Chagas, comme bien d'autres " maladies négligées », présente sur certains points, et en apparence seulement, quelques ressemblances avec le sida, elle s'en différencie en effet sur de nombreux autres qui sont essentiels.
\end{abstract}

Mots clés Maladie de Chagas - Trypanosomose · Trypanosoma cruzi $\cdot$ Amérique latine $\cdot \mathrm{VIH} \cdot \mathrm{Sida} \cdot$ Immigrants $\cdot$ Stigmatisation $\cdot$ Discrimination $\cdot$ Transmission congénitale $\cdot$ Nifurtimox $\cdot$ Benznidazole $\cdot$ Risque transfusionnel $\cdot$ Allaitement maternel $\cdot$ Abarax $^{\circledR}$

\begin{abstract}
Chagas disease and AIDS: the same terminology cannot be used to associate, let alone confuse, these two diseases with one another without distorting reality, as was done in a recent medical article entitled: Chagas disease: "The New HIV/AIDS of the Americas". Even though Chagas disease, like many other "neglected diseases", bears some superficial resemblance to AIDS in certain ways, it nevertheless differs from the latter in many other significant ones.
\end{abstract}

Keywords Chagas disease - Trypanosomiasis ·

Trypanosoma cruzi · Latin America · HIV · AIDS · Immigrants $\cdot$ Stigmatization $\cdot$ Discrimination $\cdot$ Congenital transmission $\cdot$ Nifurtimox $\cdot$ Benznidazole $\cdot$ Tranfusional risk $\cdot$ Breastfeeding $\cdot$ Abarax $^{\circledR}$

\section{J.-F. Pays $(\bowtie)$}

Faculté de médecine René-Descartes-Necker, université Paris-V-René-Descartes, 156, rue de Vaugirard, F-75015 Paris, France

e-mail : pays@necker.fr

Universidad Nacional del Nordeste (UNNE) - Argentina, Centre national des maladies parasitaires et tropicales (Cenpetrop), Corrientes, Argentine

\section{Introduction}

Une équipe nord-américaine a publié récemment dans le PLoS Neglected Tropical Diseases un article intitulé Chagas disease: "The New HIV/AIDS of the Americas" [15]. Ce titre provocateur, pour ne pas dire racoleur, ne nous paraît pas justifié et nous voulons dire ici pourquoi. En effet, si la maladie de Chagas, comme bien d'autres «maladies négligées », présente sur certains points quelques ressemblances avec le sida, elle s'en différencie sur de nombreux autres qui sont essentiels et que les auteurs minimisent ou passent sous silence.

Tout d'abord, la maladie de Chagas n'est pas une nouvelle maladie comme le sous-entend l'intitulé dont il est question. La présence de son agent, Trypanosoma cruzi, dans les tissus momifiés des Indiens Chinchorros du désert d'Atacama, quelque 9000 ans avant sa découverte par le Brésilien Carlos Chagas, en 1909 [7], a même fait de cette trypanosomose une des maladies les plus anciennes scientifiquement attestées [2], contrairement au sida qui n'est entré dans la nosographie que dans les années 1980, et dont l'existence ne dépasserait pas, dans l'hypothèse la plus extrême, une centaine d'années [36].

En second lieu, la maladie de Chagas est une maladie parasitaire endémique à transmission essentiellement vectorielle. Elle n'existe que sur le continent américain où elle se présente comme l'avatar d'une zoonose sévissant probablement depuis plusieurs millions d'années chez les très nombreuses espèces animales qui lui servent de réservoir et qui continueront, quoi qu'il advienne, à assurer sa pérennité. La trypanosomose humaine américaine à $T$. cruzi ou maladie de Chagas [26] n'a donc rien de commun sur ces différents plans avec le sida, maladie virale contagieuse pandémique qui, bien que née du franchissement accidentel de la barrière interspécifique entre homme et singe, n'a pas de véritable réservoir animal.

Ces données élémentaires et fondamentales ne sont pas rappelées avec suffisamment de force dans l'article de Hotez et al. [15] qui, a contrario, met en avant cinq traits de « ressemblance frappante » entre sida et maladie de Chagas : la présence dans ces deux maladies d'une phase aiguë, d'une 
phase de latence et d'une phase chronique, la possibilité, pour leur agent respectif, d'être transmis de la mère à son enfant ou par le sang, la nécessité, pour le sida comme pour la maladie de Chagas, d'un traitement long, coûteux et difficilement accessible, et le fait que ces deux affections, surtout si on compare la maladie de Chagas au sida des années 1980-1990, sont deux maladies touchant électivement des populations pauvres, n'ayant que très difficilement accès aux structures de soins et donnant lieu à stigmatisation et discriminations. Qu'en est-il exactement?

\section{Pauvreté, stigmatisation et discriminations}

Dès sa découverte, et bien avant que le concept de maladie négligée [17] soit inventé par l'OMS, la maladie de Chagas a été considérée comme une maladie intimement liée à la pauvreté, à l'insalubrité des habitats, à l'ignorance et à l'arriération des populations qui en étaient victimes. Elle fut même un temps rendue responsable de crétinisme en raison d'un lien erroné établi par Chagas entre goitre endémique et trypanosomose à T. cruzi [25]. Cette connotation péjorative de l'affection perdura, tant et si bien que de nombreux pays d'Amérique latine n'acceptèrent qu'avec réticence, le moment venu, d'être reconnus pays d'endémie, tant ils s'en sentaient dévalorisés. Aujourd'hui encore, des individus ou groupes d'individus refusent l'idée même d'être infectés, ainsi que le dépistage qui leur est proposé, tant ils craignent d'être déclassés en cas de résultat positif. D'autres ne font pas appel aux équipes de désinsectisation lorsqu'ils s'aperçoivent que leur maison est infestée de triatomes, punaises vecteurs de la maladie, de peur d'être montrés du doigt par les membres de leur communauté.

À l'inverse, le sida n'est pas à proprement parler une maladie de la pauvreté puisqu'il touche indifféremment riches et démunis sur tous les continents. La vraie différence entre ces deux catégories de victimes réside dans le fait que les riches, pendant les 20 premières années de la pandémie, ont bénéficié du progrès constant des connaissances, puis des thérapies, mises au point dans les pays nantis, ainsi que d'un environnement plus favorable à leur survie, alors que les populations démunies restaient souvent dans l'ignorance des risques encourus et ne pouvaient accéder aux traitements faute de moyens financiers et d'infrastructures sanitaires suffisantes. La pauvreté ainsi que l'ignorance des modes de transmission qui lui est presque toujours associée ont joué et jouent donc un rôle important dans l'épidémiologie des deux affections, comme du reste dans celle de bien d'autres maladies négligées, mais de manière très différente. Quant à la stigmatisation, qui a touché et touche les sidéens, elle était et reste d'une tout autre nature que celle qui touche les chagasiques. Elle a été d'abord liée à l'orientation sexuelle des premières victimes, puis à une peur irraisonnée de la conta- gion, faisant du sidéen, pour le peu de temps qui lui restait à vivre, le lépreux ou le pestiféré du $\mathrm{xx}^{\mathrm{e}}$ siècle.

On retrouve de nos jours des traces de cette peur dans les restrictions dont les sidéens sont victimes lorsqu'ils veulent obtenir un visa ou résider dans un des 48 pays qui pratiquent encore un tel type de discrimination, en violation de la Convention des droits de l'homme. Il n'existe, bien entendu, rien de tel pour les chagasiques qui peuvent circuler et résider dans le monde entier comme bon leur semble, mais il est vrai que d'autres formes de discrimination peuvent les concerner. Il n'est pas rare en effet, dans certains pays d'endémie, qu'un employeur exige d'un candidat à l'embauche la présentation d'un certificat attestant sa séronégativité pour la maladie de Chagas de crainte qu'un séropositif soit moins apte au travail, plus souvent absent pour raisons de santé, ou développe, au cours de son emploi, une cardiopathie évolutive que l'employeur pourrait alors être appelé à prendre partiellement en charge. Dans d'autres cas, lorsque le diagnostic est posé après l'embauche, le travailleur, le plus souvent non syndiqué, est tout simplement licencié [12].

L'exportation de cas de plus en plus nombreux [30] de maladie de Chagas hors de l'Amérique latine, tout en constituant un problème de santé publique à ne pas négliger, et qu'il faut apprendre à gérer $[4,16]$, ne constitue pas une menace pour les populations d'accueil, qu'elles soient européennes, américaines, australiennes ou autres, à partir du moment où les mesures de prévention nécessaires sont prises au niveau des centres de transfusion sanguine et des services de transplantation. Elle ne doit donc pas être présentée de façon à risquer d'être interprétée comme telle.

Quant à la discrimination dont les chagasiques feraient l'objet dans les pays non endémiques concernant l'accès à une prise en charge de leur maladie, elle est liée avant tout à leur statut d'immigré la plupart du temps clandestin qui leur interdit par prudence de se manifester et de se faire prendre en charge [19], sauf en cas de complications cardiaques majeures. Ce type de discrimination ne saurait être mis sur le même plan que celle que subissaient les sidéens des années 1980 en raison de la connotation sexuelle très particulière du sida au début de la pandémie.

\section{Traitements longs, coûteux et difficilement accessibles}

Le « traitement » du sida dure toute la vie. Il ne guérit pas encore, mais ralentit considérablement l'évolution de la maladie tout en permettant aux patients d'avoir une existence relativement proche de la normale. Sa mise au point et sa généralisation n'ont été rendues possibles que par un effort sans précédent de la recherche effectué par les pays nantis directement concernés par la pandémie, doublé par la suite, sous la pression des organisations humanitaires et 
facilité par l'effondrement du prix des antirétroviraux entre 2000 et 2002, d'un effort financier également sans précédent pour faire bénéficier de ce traitement les populations démunies.

Il en va tout différemment du traitement de la trypanosomose humaine américaine à $T$. cruzi. Nifurtimox et benznidazole guérissent définitivement $80 \%$ environ des chagasiques en phase aiguë au prix d'un certain nombre d'effets secondaires qui peuvent être sévères et nécessiter l'arrêt du traitement. Par contre, ces deux molécules ont été jugées pendant longtemps sans effet sur l'infection en phase chronique (phase indéterminée et complications tardives), et ce n'est qu'en 1990 qu'une activité leur a été reconnue dans cette dernière indication chez les enfants de moins de 12 ans $[9,32]$. Par la suite, quelques études ont, de manière plus ou moins discutable, mis en évidence, chez certains adultes traités, une évolution plus lente de la maladie, avec une entrée éventuelle plus tardive dans la phase des complications et une moindre gravité de la myocardite chronique une fois celle-ci avérée [35]. De nombreuses autres études ont suivi, dans des conditions et avec des protocoles si différents qu'il est très difficile de les comparer dans leur ensemble. Les méta-analyses les plus récentes font état, à leur sujet, de résultats contradictoires et de toute manière beaucoup trop insuffisants pour recommander un traitement de tous les chagasiques chroniques [11,20,27]. L'une d'entre elles évalue même à 5,9\% seulement le taux de guérison parasitologique des patients âgés de 15 à 70 ans traités tardivement [13]. On est donc très loin de l'efficacité de la trithérapie antirétrovirale prescrite au cours du sida. C'est une des raisons pour laquelle les décideurs des pays endémiques continuent de privilégier la lutte antivectorielle ainsi que la prévention de tous les autres modes de transmission, et se montrent réservés vis-à-vis d'un traitement qui a beaucoup de mal à faire ses preuves et concernerait les neuf à dix millions de chagasiques chroniques que compte encore le souscontinent latino-américain. Par contre, il existe aujourd'hui une manière de consensus pour traiter tous les séropositifs âgés de moins de 18 ans, avec des résultats qui sont extrêmement variables d'une région à l'autre [38]. Seule la mise au point d'un test permettant, dans un délai raisonnable, d'affirmer la guérison serait susceptible de faire bouger les lignes dans ce domaine. Pour l'heure, en effet, la guérison ne peut vraiment être affirmée, du moins en théorie, que par une séroréversion stable pendant cinq ans, et qui ne se produit généralement que très tardivement (18 mois à 15 ans après le traitement, sans parler, en zone d'endémie, des possibilités de réinfection). Cette mise au point est une priorité, mais la tâche est rude. L'existence de ce test aurait également pour effet de « booster » la recherche de nouvelles molécules plus actives et mieux tolérées. Contrairement à ce qu'on a l'habitude de laisser entendre, celle-ci n'a jamais cessé [1], mais elle n'a pas donné de résultat concret jusqu'à présent et elle n'est guère encouragée par la diminution spectaculaire de l'incidence et de la prévalence de la maladie enregistrées ces 20 dernières années suite à la mise en place des programmes de lutte antivectorielle $[10,37]$.

Les difficultés d'accès au nifurtimox et au benznidazole sont paradoxales et à l'origine d'une situation à la fois dramatique et «ubuesque ». Les solutions pour en sortir devraient donc relever du simple bon sens. Cela n'a pas été le cas jusqu'à présent et certaines des campagnes de traitement en cours ont même dû être interrompues par manque de médicament [21]. La licence de fabrication du benznidazole était détenue jusqu'à ces derniers mois par l'État brésilien d'Acre, associé au laboratoire pharmaceutique de l'État de Pernambuco (LAFEPE) qui sous-traitait la fabrication de la matière première au laboratoire privé Nortec Quimica, le tout sous l'égide du ministère de la Santé brésilien. Pour des raisons qui ne sont pas très claires, ces différentes entités se sont révélées jusqu'à présent incapables de se coordonner pour tenir leurs engagements et assurer non seulement une production régulière et suffisante de médicament, en réponse à une demande croissante, mais également sa distribution [23]. La situation est aujourd'hui en voie d'amélioration avec l'entrée en scène de deux laboratoires argentins (Maprimed et Elea) qui se sont associés pour produire, sous l'égide du ministère de la Santé argentin et de la fondation Mundo sano présidée par la propriétaire d'Elea, un générique du benznidazole sous le nom d'Abarax ${ }^{\circledR}$ (comprimés à $100 \mathrm{mg}$ et comprimés à $50 \mathrm{mg}$ facilement quadrisécables et dispersibles pour usage pédiatrique) $[18,22]$. Parallèlement, le Brésil se dit prêt à réalimenter le marché et à commercialiser également une forme pédiatrique de benznidazole, mais la production aux normes et l'enregistrement de ces comprimés dosés à $12,5 \mathrm{mg}$ ne sont pas encore assurés. Le cas du nifurtimox est différent, mais tout aussi paradoxal. Cette molécule est réputée à juste titre avoir une activité proche de celle du benznidazole, bien qu'il n'existe aucune étude comparative d'envergure entre les deux trypanocides, notamment en fonction des différentes souches de trypanosome. Malgré cela, le nifurtimox n'est pratiquement pas utilisé par les médecins sud-américains qui lui préfèrent, souvent sans arguments bien convaincants, le benznidazole, même lorsque celui-ci est introuvable ! En 2003, le laboratoire Bayer, fabricant du nifurtimox, avait pourtant mis gratuitement à la disposition de la plupart des pays endémiques plusieurs centaines de milliers de comprimés qui n'avaient pratiquement pas été utilisés [29].

La durée du traitement par le benznidazole comme par le nifurtimox est en moyenne de deux mois, ce qui n'est pas vraiment long comparé au traitement à vie du sida, ou même à un traitement de 6 à 12 mois pour une mycobactériose. Le prix d'une cure de benznidazole, lorsqu'on peut se procurer ce médicament, peut varier dans de très larges proportions (entre 50 à 600 USD et même davantage, semble-t-il) en 
fonction des pays, du degré de pénurie et du secteur : privé, publique, humanitaire... Le renchérissement prévu des produits de base nécessaires à la fabrication de la molécule ne devrait pas avoir d'incidence sur le prix de vente de l'Aba$\operatorname{rax}^{\circledR}$ fixé à 120 USD pour une cure/adulte, si l'on en croit la promesse des fabricants (J. Jannin, communication personnelle). Même si cela n'était pas tout à fait le cas, le prix d'une cure de benznidazole resterait toujours de beaucoup inférieur à celui d'une trithérapie antirétrovirale à vie. Quant au nifurtimox, également utilisé pour le traitement de la maladie du sommeil en phase de polarisation cérébrale, il peut toujours être obtenu gratuitement auprès de l'OMS grâce à une dotation sur le long terme du laboratoire Bayer.

La prise en charge des complications cardiaques et digestives de la maladie de Chagas coûte évidemment beaucoup plus cher que son traitement étiologique. Entre 1992 et 2000, 1140 millions d'USD auraient été dépensés par les seuls pays du Cône Sud en traitements symptomatiques, soins palliatifs et nombre d'années de travail et de vie perdues (DALYs).

C'est même ce coût, à la fois réel et théorique, qui a motivé en grande partie l'implication des états latino-américains dans les grandes initiatives régionales de lutte prises entre 1991 et 1997 visant à éliminer la transmission vectorielle de la maladie, avec, tout au moins sur le papier, la quasi-certitude d'un retour sur investissement très rapide [31].

\section{Mode d'évolution des deux maladies}

En l'absence de traitement, c'est probablement dans la façon dont évoluent ces deux affections que le sida et la maladie de Chagas présentent le plus de fausses ressemblances. Toutes deux commencent en effet par une phase aiguë habituellement paucisymptomatique passant souvent inaperçue. Toutefois, la maladie de Chagas peut se compliquer dès les premières semaines chez le jeune enfant, victime privilégiée de la transmission vectorielle, d'une myocardite aiguë ou d'une méningoencéphalite mortelles et, chez l'enfant comme chez l'adulte, se manifester par des chagomes assez caractéristiques, ou encore par un œdème violacé de l'œil, unilatéral, bipalpébral, pathognomonique, appelé signe de Romaña. La phase de latence qui succède à la phase aiguë est d'une durée extrêmement variable dans les deux affections. Elle se termine, dans moins d'un cas sur trois de maladie de Chagas, par des complications tardives de gravité variable sanctionnées par une mortalité annuelle de $1 \%$ à $1,5 \%$ et, dans quasiment tous les cas de sida, par une immunodépression profonde toujours fatale à plus ou moins brève échéance. Dans le premier cas, il s'agit bien de complications. Dans le second, il s'agit de l'évolution naturelle de la maladie.

\section{Transmission congénitale}

La transmission congénitale du sida concerne, dans les pays en développement, 25 à $45 \%$ des enfants nés de mères infectées. On regroupe habituellement sous ce terme la transmission in utero, la transmission au cours de l'accouchement et, un peu abusivement, la transmission par l'allaitement qui représente un peu moins de la moitié des cas de transmissions mère-enfant. Un traitement préventif bien conduit abaisse la fréquence de ces transmissions à moins de $5 \%$ et pourrait théoriquement la ramener à des valeurs proches de zéro [34]. À l'opposé, $5 \%$ seulement des mères chagasiques transmettent la maladie à leur enfant in utero. Par contre, il n'existe pas de traitement préventif mais l'enfant, s'il est infecté, peut être définitivement guéri dans pratiquement $100 \%$ des cas s'il est traité avant l'âge de six mois, et sans effet secondaire si le traitement a lieu dès les premières semaines de vie. Ensuite, l'efficacité du traitement et la tolérance décroissent au fil des mois et des années [6]. Le dépistage des femmes en âge de procréer et des femmes enceintes, en et hors pays d'endémie, est donc un immense défi qui doit être relevé et gagné si l'on veut que la maladie de Chagas appartienne un jour au passé.

\section{La voie orale}

La transmission de T. cruzi par le lait maternel, malgré quelques soupçons, n'a jamais été prouvée. Il n'existe donc aucune restriction à ce qu'une mère chagasique nourrisse son enfant. On vient de dire l'importance du rôle de l'allaitement dans la transmission du VIH. Laisser entendre que « l'absence de transmission par voie orale » pour le sida est l'un des trois principaux points qui différencient le sida de la maladie de Chagas [15] est donc inexact. Les deux maladies peuvent bel et bien se transmettre par voie orale, mais uniquement par le lait maternel pour le sida et, apparemment, jamais de cette manière pour la maladie de Chagas, mais par l'intermédiaire d'aliments ou de boissons souillés par des déjections de triatomes infectés, par l'urine de certains animaux réservoirs comme les opossums, ou encore par des broyats d'insectes infectés accidentellement introduits dans les boissons ou la nourriture $[8,33]$. Les maladies de Chagas contractées de cette manière revêtent une gravité particulière chez l'enfant comme chez l'adulte.

Pour renforcer probablement, auprès du grand public, l'idée que la maladie de Chagas restait bien l'objet de toutes les négligences, il n'est pas dit un seul mot [15] des très nombreux travaux de recherche menés sur la trypanosomose humaine américaine à $T$. cruzi depuis le début du siècle dans la plupart des pays endémiques, et ailleurs. Chaque jour pourtant, et cela depuis plusieurs dizaines d'années, ces travaux donnent lieu à une bonne douzaine de publications 
scientifiques de niveau international. De même, ne sont ni rappelées ni même citées les grandes initiatives régionales (INCOSUR pour les pays du Cône Sud, IPA pour les pays du Pacte andin, IPCA pour les pays d'Amérique centrale) prises successivement, sous l'égide de la PAHO, pour contrôler, voire éliminer, la transmission vectorielle $[14,24,28,37]$. Pourtant, grâce à ces initiatives, la prévalence de la maladie a été réduite en moins de 20 ans de plus de $50 \%$ et son incidence de pratiquement $90 \%$ [26]. Même s'il reste encore beaucoup à faire, notamment au niveau de la détection précoce et du traitement des nouveaux cas, qu'ils soient congénitaux ou acquis, il n'y a pas de raison pour que ces succès ne soient pas signalés et salués. Présenter enfin la maladie de Chagas comme une maladie oubliée que l'on redécouvre brusquement, c'est faire bon marché du dévouement et du travail des médecins, des chercheurs, du personnel infirmier et des agents de santé publique sud-américains qui s'y consacrent quotidiennement depuis un siècle, avec ou sans l'aide des ONG.

Comme on pouvait s'y attendre avec un pareil titre, Chagas disease: "the new HIV/AIDS of the Americas" a trouvé dans les médias, dont le très sérieux New York Times, un écho inhabituel pour un article paru dans un journal de médecine, et ce, en raison du très grand nombre de chagasiques chroniques qui vivent aujourd'hui en Amérique du Nord et qui sont présentés, avec une certaine ambiguité, comme un danger potentiel, bien qu'un contrôle strict soit effectif au niveau des banques de sang nord-américaines depuis 2006 [4]. Des centaines de sites Internet, à leur suite, ont repris le titre en question et s'y réfèrent, la plupart en forçant le trait souvent jusqu'à la caricature, parlant par exemple de «maladie qui fait exploser le cœur et même les reins » [3], ou du nombre très restreint de personnes pouvant être traitées en raison du coût élevé des médicaments, ou encore d'une nouvelle variante du sida découverte récemment par les chercheurs américains... La consultation d'Internet, en associant sida/maladie de Chagas, est instructive sur le sujet.

Au moment même où un autre syndrome d'immunodépression, proche du sida dans sa traduction clinique, mais différent par son mécanisme physiopathologique comme dans son épidémiologie, est en train d'être individualisé et caractérisé en Asie [5], l'heure n'est pas aux amalgames et aux comparaisons contestables et douteuses qui ne peuvent générer, dans le grand public, qu'inquiétudes et confusions.

Sida et maladie de Chagas sont deux affections qui diffèrent fondamentalement l'une de l'autre et qui ne présentent, en fait, qu'un certain nombre de fausses ressemblances au niveau de leurs modes accessoires de transmission, de leur mode d'évolution, des problèmes posés par leur prise en charge et des caractéristiques socio-économiques des populations qu'elles ciblent.
Non, même pour la « bonne cause » et l'efficacité de la communication - puisque c'est bien de cela dont on espère qu'il s'agit - la maladie de Chagas ne peut être présentée sous les oripeaux du sida, cette affection si tragiquement connotée et référencée aux fantasmes de la déviance, du sexe et de la mort.

\section{Références}

1. Apt W (2010) Current and developing therapeutics agents in the treatment of Chagas disease. Drug Des Devel Ther 4:243-53

2. Aufderheide AC, Salo W, Madden M, et al (2004) A 9000-year record of Chagas' disease. Proc Natl Acad Sci U S A 101 (7):2034-9. Epub 2004 Feb 6

3. Belge Info (2012) La maladie de Chagas est le nouveau sida des Amériques [www.belgeinfo.com/index.php/328]

4. Bern C, Montgomery SP, Herwaldt BL, et al (2007) Evaluation and treatment of Chagas disease in the United States: a systematic review. JAMA 298(18):2171-81

5. Browne SK, Burbelo PD, Chetchotisakd P, et al (2012) Adultonset immunodeficiency in Thailand and Taiwan. N Eng J Med 367(8):725-34

6. Brutus L, Santalla JA, Salas NA, et al (2009) Le dépistage de l'infection congénitale par Trypanosoma cruzi en France métropolitaine. Bull Soc Pathol Exot 102(5):300-10 [http://www. pathexo.fr/documents/articles-bull/T102-5-300-309.pdf]

7. Chagas C (1909) Nouvelle espèce de trypanosomiase humaine. Bull Soc Pathol Exot 2(6):304-7 [http://www.pathexo.fr/documents/articles-bull/T2-6-304.pdf]

8. Coura JR (2006) Transmissao da infeçao chagasica por via oral na historia natural da doença de Chagas. Rev Soc Bras Med Trop 39(SIII):113-7

9. de Andrade AL, de Zicker F, de Oliveira RM, et al (1996) Randomised trial of efficacy of benznidazole in treatment of early Trypanosoma cruzi infection. Lancet 348(9039):1407-13

10. Dias JC, Silveira AC, Schofield CJ (2002) The impact of Chagas disease control in Latin America: a review. Mem Inst Oswaldo Cruz 97(5):603-12

11. Fuentes RB, Maturana AM, de la Cruz MR (2012) Eficacia de nifurtimox para el tratamiento de pacientes con enfermedad de Chagas cronica. Rev Chilena Infectol 29(1):82-6. Epub 2012 Apr 10

12. Guariento ME, Camilo MV, Camargo AM (1999) Working conditions of Chagas' disease patients in a large Brazilian city. Cad Saude Publica 15(2) 381-6

13. Guedes PM, Silva GK, Gutierrez FR, Silva JS (2011) Current status of Chagas disease chemotherapy. Expert Rev Anti Infect Ther 9(5):609-20

14. Hashimoto K, Schofield CJ (2012) Elimination of Rhodnius prolixus in Central America. Parasit Vectors 5:45

15. Hotez PJ, Dumonteil E, Woc-Colburn L, et al (2012) Chagas disease: "The New HIV/AIDS of the Americas". PLoS Negl Trop Dis 6 6(5):e1498. Epub 2012 May 29

16. Jackson Y, Angheben A, Carrilero Fernandez B, et al (2009) Prise en charge de la maladie de Chagas en Europe. Expériences et défis en Espagne, Suisse et Italie. Bull Soc Pathol Exot 102(5) 326-29 [http://www.pathexo.fr/documents/articles-bull/T102-5326-329.pdf]

17. Jannin J, Simarro PP, Louis FJ (2003) Le concept de maladie négligée. Med Trop 63(3):219-21

18. Laboratorio Elea (2012) Abarax ${ }^{\circledR}$ contra la enfermedad olvidada [www.elea.com/abarax.php] 
19. Lescure FX, Paris L, Elghouzzi MH, et al (2009) Expérience de dépistage ciblé de la maladie de Chagas en Île-de-France. Bull Soc Pathol Exot 102(5):295-99 [http://www.pathexo.fr/documents/articles-bull/T102-5-295-299.pdf]

20. Matta Guedes PM, Gutierrez FR, Nascimento MS (2012) Antiparasitical chemotherapy in Chagas' disease cardiomyopathy: current evidence. Trop Med Int Health 17(9):1057-65. Epub 2012 Jun 12

21. Médecins sans frontières, Access Campaign (2011) Treatment ends for Chagas patients [www.msfaccess.org/about-us/mediaroom/press-releases/treatment-ends-chagas-patients]

22. Médecins sans frontières, Access Campaign (2012) Argentina joins Brazil in producing life-saving drug for neglected disease [www.msfaccess.org/about-us/media-room/press-releases/argentina-joins-brazil-producing-life-saving-drug-neglected]

23. Médecins sans frontières, Access Campaign (2012) Critical shortage of first-line therapy for Chagas: The story of benznidazole [www.msfaccess.org/content/critical-shortage-first-line-therapychagas-story-benznidazole]

24. Pan America Health Organization (2012) INCOSUR-Chagas: Southern Cone Initiative to Control/Eliminate Chagas Disease [www.paho.org/english/ad/dpc/cd/incosur.htm]

25. Pays JF (2009) Chagas Carlos Justiniano Ribeiro (1879-1934). Bull Soc Pathol Exot 102(5):276-9 [http://www.pathexo.fr/documents/articles-bull/T102-5-276-279.pdf]

26. Pays JF (2009). Entre épée de Damoclès et roulette russe... ce que tout le monde sait ou devrait savoir sur la maladie de Chagas. Bull Soc Pathol Exot 102(5):280-84 [http://www.pathexo.fr/ documents/articles-bull/T102-5-280-284.pdf]

27. Pérez-Molina JA, Pérez-Ayala A, Moreno S, et al (2009) Use of benznidazole to treat chronic Chagas' disease: a systematic review with a meta-analysis. J Antimicrob Chemother 64(6):1139-47. Epub 2009 Oct 9
28. Ponce C (2007) Current situation of Chagas disease in Central America. Mem Inst Oswaldo Cruz 102(Suppl 1):41-4. Epub 2007 Nov 5

29. Ruiz JA, Karunakara U (2006) Chagas disease update on progress and future perspectives. Médecins sans frontières, Access Campaign [www.ebookbrowse.com/negdis-report-chagas-updateonprogress-eng-2006-pdf-d247372018]

30. Schmunis GA, Yadon ZE (2010) Chagas disease: a Latin American health problem becoming a world health problem. Acta Trop 115(1-2):14-21. Epub 2009 Nov 20

31. Schofield CJ, Dias JC (1991) A cost-benefit analysis of Chagas disease control. Mem Inst Oswaldo Cruz 86(3):285-95

32. Sosa Estani S, Segura EL, Ruiz AM, et al (1998) Efficacy of chemotherapy with benznidazole in children in the indeterminate phase of Chagas' disease. Am J Trop Med Hyg 59(4):526-9

33. Toso MA, Vial UF, Galanti N (2011) Transmisión de la enfermedad de Chagas por vía oral. Rev Med Chil 139(2)258-66. Epub 2011 Jul 11

34. Van de Perre P, Rubbo PA, Viljoen J, et al (2012) HIV-1 reservoirs in breast milk and challenges to elimination of breastfeeding transmission of HIV-1. Sci Transl Med 4(143):143sr3

35. Viotti R, Vigliano C, Lococo B, et al (2006) Long-term cardiac outcomes of treating chronic Chagas disease with benznidazole versus no treatment: a nonrandomized trial. Ann Intern Med 144(10):724-34

36. Worobey M, Gemmel M, Teuwen DE, et al (2008) Direct evidence of extensive diversity of HIV-1 in Kinshasa by 1960. Nature 455(7213):661-4

37. Yamagata Y, Nakagawa J (2006) Control of Chagas disease. Adv Parasitol 61:129-65

38. Yun O, Lima MA, Ellman T, et al (2009) Feasibility, drug safety, and effectiveness of etiological treatment programs for Chagas disease in Honduras, Guatemala, and Bolivia: 10-year experience of Médecins sans frontières. PLoS Negl Trop Dis 3(7):e488 